\title{
TREM-1 Attenuates RIPK3-mediated Necroptosis in Hyperoxia-induced Lung Injury in Neonatal Mice
}

\author{
Mansoor Ali Syed ${ }^{1 * \ddagger}$, Dilip Shah ${ }^{1 *}$, Pragnya Das ${ }^{1}$, Sture Andersson ${ }^{2}$, Gloria Pryhuber $^{3}$, and Vineet Bhandari ${ }^{1}$ \\ ${ }^{1}$ Drexel University College of Medicine, Philadelphia, Pennsylvania; ${ }^{2}$ Children's Hospital, University of Helsinki and Helsinki University \\ Hospital, Helsinki, Finland; and ${ }^{3}$ Department of Pediatrics, University of Rochester School of Medicine and Dentistry, Rochester, New \\ York
}

ORCID IDs: 0000-0002-4248-5611 (D.S.); 0000-0002-4900-6636 (V.B.).

\begin{abstract}
Hyperoxia-induced injury to the developing lung, impaired alveolarization, and dysregulated vascularization are critical factors in the pathogenesis of bronchopulmonary dysplasia (BPD); however, mechanisms for hyperoxia-induced development of BPD are not fully known. In this study, we show that TREM-1 (triggering receptor expressed on myeloid cells 1 ) is upregulated in hyperoxiaexposed neonatal murine lungs as well as in tracheal aspirates and lungs of human neonates with respiratory distress syndrome and $\mathrm{BPD}$ as an adaptive response to survival in hyperoxia. Inhibition of TREM-1 function using an siRNA approach or deletion of the Trem 1 gene in mice showed enhanced lung inflammation, alveolar damage, and mortality of hyperoxia-exposed neonatal mice. The treatment of hyperoxia-exposed neonatal mice with agonistic TREM-1 antibody
\end{abstract}

decreased lung inflammation, improved alveolarization, and was associated with diminished necroptosis-regulating protein RIPK3 (receptor-interacting protein kinase 3). Mechanistically, we show that TREM-1 activation alleviates lung inflammation and improves alveolarization through downregulating RIPK3-mediated necroptosis and NLRP3 (nucleotide-binding oligomerization domain-like receptor containing pyrin domain 3) inflammasome activation in hyperoxia-exposed neonatal mice. These data show that activating TREM-1, enhancing angiopoietin 1 signaling, or blocking the RIPK3-mediated necroptosis pathway may be used in new therapeutic interventions to control adverse effects of hyperoxia in the development of BPD.

Keywords: TREM-1; necroptosis; NLRP3 inflammasome; hyperoxia-induced lung injury; bronchopulmonary dysplasia
Hyperoxia-induced injury to the developing lung, impaired alveolarization, and dysregulated vascularization are critical factors in the pathogenesis of bronchopulmonary dysplasia (BPD) $(1,2)$; however, mechanisms for hyperoxia (HYP)-induced development of BPD are not fully known. After hyperoxia-induced acute lung injury (HALI) or mechanical ventilation during premature birth, damage-associated molecular patterns are released from necrotic cells and recognized by pattern recognition receptors that include Toll-like receptors and nucleotidebinding oligomerization domain-like receptors (3-5). The activation of these molecules has been recognized as a modulator of inflammation and decreased alveolarization in a mouse model of BPD (3) as well as in preterm infants (3). In recent years, a new family of the innate immune receptor has been identifiedknown as triggering receptors expressed on myeloid cells (TREMs) - that have been shown to modulate immune response because of their ability to amplify or decrease the signals induced by Toll-like

(Received in original form July 3, 2018; accepted in final form October 1, 2018)

*These authors contributed equally.

${ }^{\ddagger}$ Present address: Department of Biotechnology, Jamia Millia Islamia, New Delhi, India.

Supported in part by departmental funds (M.A.S., D.S., P.D., and V.B.), National Heart, Lung, and Blood Institute grant HL63039 (G.P.) from the National Institutes of Health, and a Finnish governmental subsidy for clinical research (S.A.).

Author Contributions: Concept and design: M.A.S., D.S., and V.B.; acquisition of data: M.A.S., D.S., P.D., S.A., and G.P.; data analysis and interpretation: M.A.S., D.S., and V.B.: and drafting, editing, and/or critical revision of the manuscript for intellectual content: M.A.S., D.S., P.D., S.A., G.P., and V.B. All authors approved the submitted version of the manuscript.

Correspondence and requests for reprints should be addressed to Vineet Bhandari, M.D., D.M., Section of Neonatal-Perinatal Medicine, Department of Pediatrics, Drexel University College of Medicine, NCB, Suite \#7410, 245 N. 15th Street, Philadelphia, PA 19102. E-mail: vineet.bhandari@drexel.edu.

This article has a data supplement, which is accessible from this issue's table of contents at www.atsjournals.org.

Am J Respir Cell Mol Biol Vol 60, Iss 3, pp 308-322, Mar 2019

Copyright $\odot 2019$ by the American Thoracic Society

Originally Published in Press as 10.1165/rcmb.2018-02190C on October 3, 2018

Internet address: www.atsjournals.org 


\section{Clinical Relevance}

Hyperoxia-induced inflammation and alveolar damage are critical contributors to hyperoxia-induced acute lung injury (HALI) and the development of bronchopulmonary dysplasia (BPD), the mechanisms of which have not been fully characterized. RIPK3 (receptorinteracting protein kinase 3)-mediated necroptosis has been identified in several lung diseases, such as acute respiratory distress syndrome, chronic obstructive pulmonary disease, and sepsis. We show that TREM-1 (triggering receptor expressed on myeloid cells 1) functions by attenuating necroptosis and NLRP3 (nucleotide-binding oligomerization domain-like receptor containing pyrin domain 3) inflammation in neonatal lungs exposed to hyperoxia, and we identify TREM-1, angiopoietin 1, and RIPK3 as potential therapeutic targets for the treatment of neonatal HALI and BPD. We show that activating TREM1 , enhancing angiopoietin 1 signaling, and/or blocking the RIPK3-mediated necroptosis pathway may act as new therapeutic interventions to control the adverse effects of HALI in the development of BPD.

receptors and nucleotide-binding oligomerization domain-like receptors $(6,7)$. However, the role of TREM-1 in neonatal HALI and its contribution to the development of BPD is not known.

Our overarching hypothesis is that TREM-1 expression is required for limiting lung inflammation, alveolar injury, and survival in HYP. We report that TREM-1 expression is increased in the lungs of HYP-exposed neonatal mice as well as human neonates with respiratory distress syndrome (RDS) and BPD as an adaptive survival response. In HYP-exposed neonatal mice, we observed that deletion of the Trem 1 gene in mice leads to increased lung inflammation, alveolar damage, and mortality. We further observed that increased lung inflammation is associated with enhanced necroptosis-regulating protein RIPK3 (receptor-interacting protein kinase 3)-mediated necroptosis and NLRP3 (nucleotide-binding oligomerization domain-like receptor containing pyrin domain 3) inflammasome activation in the lungs of HYP-exposed neonatal mice and human neonates with RDS and BPD. We next tested whether TREM-1 confers protection to HYP-exposed neonatal mice by blocking necroptosis-regulating protein RIPK3-mediated necroptosis and NLRP3 inflammasome activation. The treatment of HYP-exposed neonatal mice with agonistic TREM-1 antibody decreased NLRP3 inflammasome activation, improved alveolarization, and was associated with diminished RIPK3-mediated necroptosis in the lungs of neonatal mice. We show that, mechanistically, TREM-1 alleviates pulmonary inflammation and alveolar injury by downregulating RIPK3-mediated necroptosis and NLRP3 inflammasome activation through induction of vascular endothelial growth factor A (VEGF-A) and augmenting angiopoietin 1 (Ang1) expression in lungs of HYP-exposed neonatal mice. Taken together, our data show that activating TREM-1, enhancing Ang1 signaling, or blocking RIPK3-mediated necroptosis may represent novel therapeutic targets for HALI and BPD in neonates.

\section{Methods}

Please refer to the data supplement for details regarding the materials and methods used in this work.

\section{Human Lung Tracheal Aspirates}

The collection and processing of the lung tracheal aspirates (TA) from premature infants being mechanically ventilated in the first postnatal week with an indwelling endotracheal tube were approved by the human investigation committee (institutional review board) of Yale University, and was done after obtaining consent was obtained from one or both parents (V.B.). Selected clinical details are provided in Table E1 in the data supplement.

\section{Animals}

All breeding pairs of the wild-type (WT) laboratory mice of the C57BL/6J strain were purchased from The Jackson Laboratory, and breeding pairs of mice with targeted deletion of TREM-1/3 and RIPK3 genes on a C57BL/6J background were obtained from Genentech. These null mutant mice have been characterized previously $(8,9)$.
All mice were housed and bred in Drexel University animal care facilities and allowed free access to standard food and water. All animal protocols were reviewed and approved by the institutional animal care and use committees of Drexel University before any studies were performed.

\section{Neonatal Mouse Model of HAL}

Newborn mice were used in all studies, and litter sizes for each experiment were adjusted to 8-10 pups per treatment group to minimize the effects of differences in nutrition on lung development. For the HALI model, newborn WT, TREM-1/3-1and RIPK3 $3^{-l-}$ mice were exposed to either room air $\left(\mathrm{RA} ; 21 \% \mathrm{O}_{2}\right)$ or HYP $\left(60 \% \mathrm{O}_{2}\right)$ continuously from Postnatal Days 1-7 (PN1-PN7) as previously described (10).

\section{Immunohistochemistry in Human Neonatal Lungs}

See the Supplemental Methods section in the data supplement for details.

\section{Analysis of BAL Fluid}

BAL was performed as described previously (11).

\section{Alveolar Macrophage Preparation from BAL Fluid}

See the Supplemental Methods section in the data supplement for details.

\section{Cell Culture and Reagents}

Murine macrophage-like RAW 264.7 cells (TIB-71; American Type Culture Collection) were exposed to HYP in sealed, humidified chambers flushed with $85 \%$ $\mathrm{O}_{2} / 5 \% \mathrm{CO}_{2}$ at $37^{\circ} \mathrm{C}$ as previously described (10). After experimental time points, cells were scraped off with a sterile cell scraper and stored in RNAlater stabilization solution (Thermo Fisher Scientific) for RNA isolation and cell lysis buffer for Western blot analysis.

\section{ELISA}

Cytokines (TNF- $\alpha$, IL-6, and IL-1 $\beta$ ) and lung myeloperoxidase concentrations were quantified using commercially available DuoSet ELISA kits (R\&D Systems) according to the manufacturer's instructions, as previously described (12).

\section{Western Blot Analysis}

Western blot analysis was performed as described previously (13). 


\section{Lung Morphometric Analysis}

At PN7, six or seven random images per lung and six lungs per experimental group were characterized for measuring lung morphometric analysis (Image-Pro Plus 4.0; Media Cybernetics). Alveolar size was estimated from the mean chord length of the airspace and radial alveolar count, as described previously (10).

\section{Intranasal Delivery of TREM-1 siRNA}

TREM-1 siRNA was procured from Ambion and reconstituted to $20 \mu \mathrm{M}$, following the manufacturer's instructions. Briefly, pups were exposed to $60 \% \mathrm{O}_{2}$ immediately after birth following the protocol, as described by Sun and colleagues (14).

\section{RNA Extraction and PCR}

Real-time and reverse transcriptase PCRs were performed as described previously $(1,15)$.

\section{Statistical Analysis}

Statistical analysis was performed using Prism 7.0 software (GraphPad Software). Two-group comparisons were analyzed by unpaired Student's $t$ test, and multiplegroup comparisons were performed using one-way ANOVA followed by Tukey's post hoc analysis. Statistical significance was achieved with $P<0.05$.

\section{Results}

\section{HYP Induces TREM-1 Expression Localized to Alveolar Macrophages in Neonatal Murine Lungs}

To determine the contribution of TREM-1 to HALI, we first examined the expression of TREM-1 and its localization in the lung compartments of RA $\left(21 \% \mathrm{O}_{2}\right)$ - or HYP $\left(60 \% \mathrm{O}_{2}\right)$-exposed neonatal mice. We selected $60 \% \mathrm{O}_{2}$, as opposed to $80-100 \%$ $\mathrm{O}_{2}$, to more closely mimic the supplemental $\mathrm{O}_{2}$ exposure as currently used for human neonates, predisposing them to developing the more common moderate BPD versus the severe BPD phenotype (16). As shown in Figures $1 \mathrm{~A}$ and $1 \mathrm{~B}$, the transcript and protein expression of TREM-1 was significantly higher in the lungs of HYPexposed neonatal mice than in RA-exposed littermates at PN7. To evaluate the constitutive presence of TREM-1 within the pulmonary compartment, cells from BAL fluid (BALF) and lungs were collected from RA- or HYP-exposed neonatal mice at PN7 for flow cytometric or confocal microscopic analysis, respectively. As shown in Figures E1A and E1B, the expression of TREM-1 on alveolar macrophages (AM) (CD11c-positive cells) was higher in cells isolated from BALF as well as in lung tissue of HYP-exposed neonatal mice than in the RA group. We saw minimal colocalization with endothelial cells for TREM-1 in neonatal murine lungs (Figure E1B).

To further confirm the induction and localization of TREM-1 by AM, we isolated AM from BALF of RA- and HYP-exposed neonatal mice at PN7 and measured transcript and protein expression of TREM-1 concentrations. As shown in Figures 1C and 1D, the transcript and protein expression of TREM-1 was significantly higher in AM isolated from neonatal mice exposed to HYP than in the RA group. To corroborate these in vivo findings, we exposed murine macrophagelike RAW 264.7 cells to RA or HYP for various times and measured the transcript and protein concentrations of TREM-1. As shown in Figures $1 \mathrm{E}$ and $1 \mathrm{~F}$, transcript concentrations of TREM-1 were elevated in a time-dependent manner in murine macrophage-like RAW 264.7 cells exposed to HYP. Taken together, these results suggest that TREM-1 expression is enhanced in response to HYP and is localized to AM in the developing lung.

\section{TREM-1 Expression Is Upregulated in Human Neonatal Lungs with RDS and BPD}

To ascertain the relevant potential functional role of TREM-1 in the development of BPD, we assessed the relative expression of TREM-1 in the TA and lung tissues of human neonates with RDS and BPD. We found that the transcript concentration of TREM-1 was significantly higher in the TA of infants who subsequently developed $\mathrm{BPD} /$ died than in those who did not develop BPD (Figure 1G). The detailed characterizations of enrolled infants are shown in Table E1. We next measured TREM-1 protein concentration in the lungs of human neonates at various stages of BPD development. We found that the protein concentration of TREM-1 was significantly higher in lungs of neonates with RDS and BPD than in term control babies (Figure 1H). The detailed characterization of this second independent cohort of enrolled infants is shown in Table E2. Finally, we confirmed the increased concentration of TREM-1 in lungs of neonates with RDS and BPD by immunohistochemical staining in a third independent cohort of human neonatal lungs with RDS and BPD (Figure 1I). The detailed characterization of enrolled infants is shown in Table E3. Altogether, these data from HYP-exposed neonatal laboratory mice and three independent cohorts of human lung samples from neonates with RDS and BPD confirmed that TREM- 1 is induced during HYP exposure in the developing lung.

\section{Suppression or Deletion of TREM-1 Gene Is Associated with Increased Pulmonary Alveolar Injury and Mortality}

To evaluate the functional role of TREM-1 in the outcome of pulmonary alveolar injury and mortality in HYPexposed neonatal mice, we first studied loss of function of TREM-1 using a siRNA approach to inhibit TREM-1 function or to completely abolish TREM-1 function using TREM-1/3-deficient mice. We selected TREM-1/3-deficient mice because previous studies have shown that TREM-1 lies adjacent to the TREM-3 gene and that these two genes are likely to have complementary functions (8). In addition, TREM-3 is a pseudogene in humans; hence, TREM-1/3deficient mice would reflect the lack of functionality of TREM-1 in humans (8). As shown in Figure 2A, TREM-1 transcript concentrations were significantly decreased (75\%) in the lungs of neonatal mice treated with TREM-1 siRNA compared with the scrambled siRNA-treated group. We next determined the effect of TREM-1 silencing in HYP-exposed neonatal mice. Surprisingly, we found that inhibition of TREM-1 led to enhanced lung inflammation as manifested by increased neutrophil recruitment (Figure 2B) and inflammatory cytokine IL-1 $\beta$ (Figure 2C) in the lungs of neonatal mice exposed to HYP. This increased lung inflammation was also allied with impaired alveolarization as demonstrated by increased chord length (Figures 2D and 2E) and decreased radial alveolar count (Figure 2F) in the lungs of TREM-1 siRNA mice exposed to HYP. These detrimental effects of TREM-1 inhibition were concomitant with decreased vascularization (Figure $2 \mathrm{G}$ ), increased apoptosis (Figure $2 \mathrm{H}$ ), and higher mortality of HYP-exposed TREM-1 siRNA neonatal mice, as compared with the scrambled siRNA mice group (Figure E2). 
A

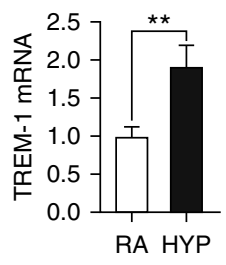

B

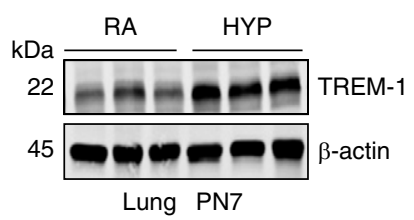

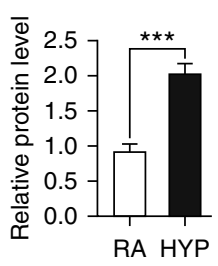

C

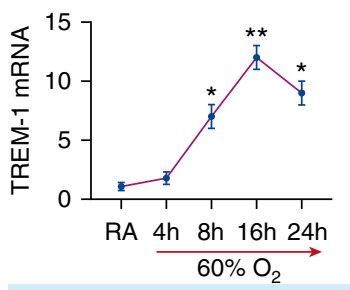

Isolated alveolar macrophages
D

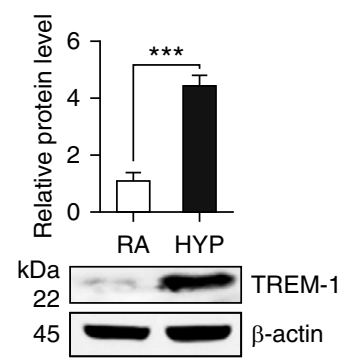

Isolated alveolar macrophages at PN7
E

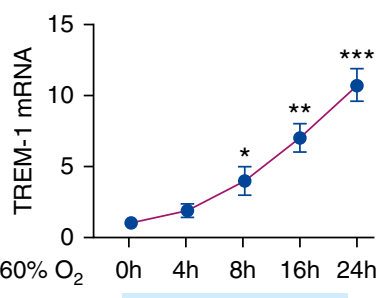

RAW 264.7 cells
$\mathbf{F}$

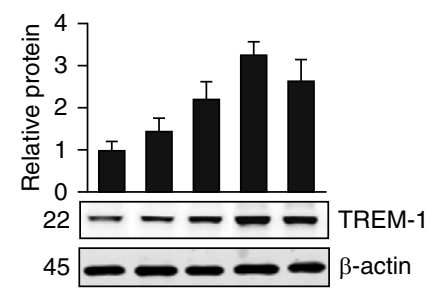

$60 \% \mathrm{O}_{2}$ Oh 4h 8h 16h 24h

RAW 264.7 cells
G

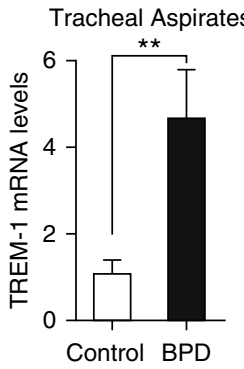

H
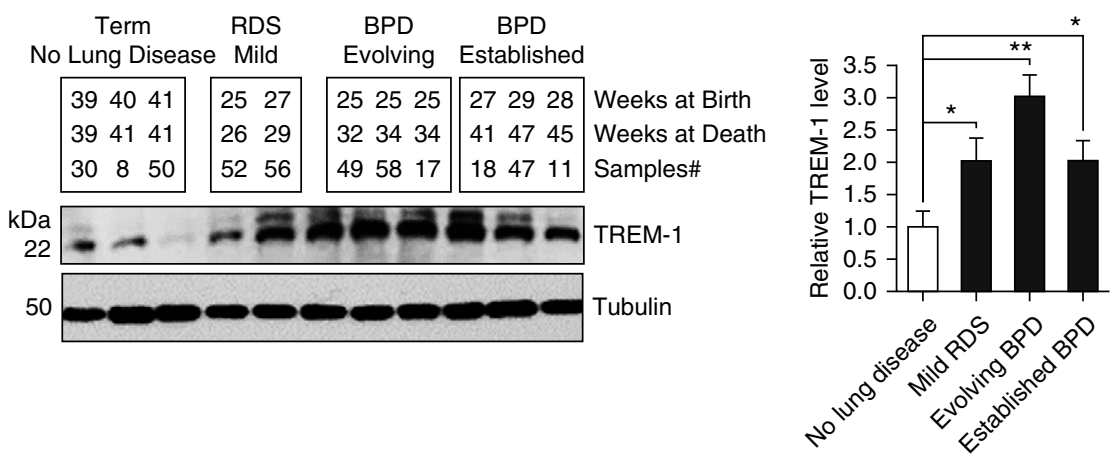

I

TREM-1 Staining in human neonatal Lung
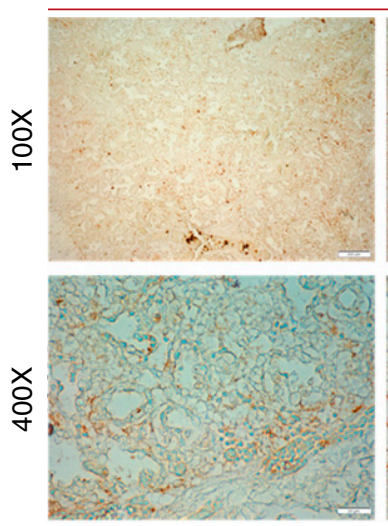

Fetus 25 weeks
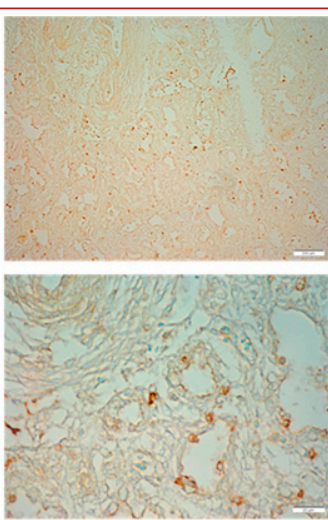

RDS 1 day
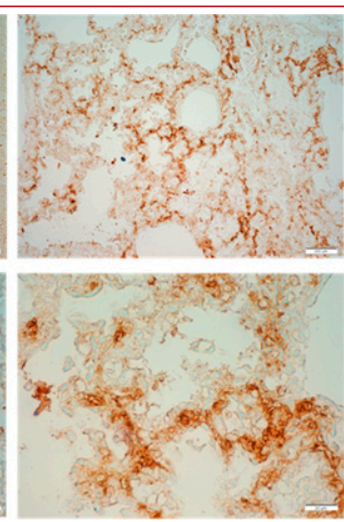

RDS 5 day

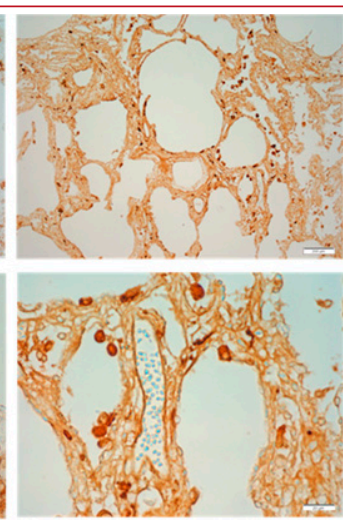

BPD

Figure 1. Triggering receptor expressed on myeloid cells 1 (TREM-1) expression is increased in the lungs of neonatal mice exposed to hyperoxia and lungs of human infants with respiratory distress syndrome (RDS) and bronchopulmonary dysplasia (BPD). (A and $B$ ) Transcript and protein expression of TREM-1 in the lungs of newborn mice exposed to hyperoxia for 7 days. (C) TREM-1 expression in alveolar macrophages isolated from neonatal mice at Postnatal Day 7 (PN7) and exposed to hyperoxia for various time points. There was no basal induction of TREM-1 in control cells. The mRNA of TREM-1 in response to hyperoxia showed a time-dependent increase up to 16 hours. (D) TREM-1 protein in the alveolar macrophages isolated from room 
To finally confirm the loss of function of TREM-1 in pulmonary inflammation and alveolar damage and the survival response to HYP, we challenged newborn TREM-1/3deficient and control WT mice to HYP or RA until PN7. Similarly to the results of TREM-1 siRNA-treated mice exposed to HYP, we found that TREM-1/3-deficient neonatal mice also showed enhanced neutrophil recruitment (Figure 2I) and myeloperoxidase concentrations (Figure 2J) in the lungs. In addition, we noted robust lung inflammation as manifested by increased inflammatory cytokines IL- 6 and IL-1 $\beta$ (Figures $2 \mathrm{~K}$ and $2 \mathrm{~L}$ ), increased alveolar damage as demonstrated by enhanced chord length, decreased radial alveolar count (Figures 2M-2O), and increased mortality in HYP-exposed TREM-1/3-deficient neonatal mice (Figure 2P). Importantly, we did not detect any changes in lung morphometry, inflammation, and mortality in TREM-1/3deficient neonatal mice exposed to RA as compared with WT littermates. These results suggest that enhanced TREM-1 expression may be essential for protecting the developing lung against HYP-induced lung inflammation, alveolar injury, and survival during HYP.

\section{TREM-1 Activation Decreases Lung Inflammation and Alveolar Injury in HYP-exposed Neonatal Mice}

Because we found that the inhibition of TREM-1 expression in lungs led to increased lung inflammation, alveolar injury, and mortality in HYP-exposed neonatal mice, we reasoned that augmenting pulmonary TREM-1 expression would attenuate pulmonary alveolar injury and improve survival. To test this hypothesis, we treated HYP- or RA-exposed neonatal mice with the TREM-1 agonist antibody by subcutaneous injection on alternate days starting at PN2 and continuing through PN6. The control group was treated similarly with an isotype antibody. Consistent with the known agonistic activity of TREM-1 antibody $(17,18)$, we found that augmentation of TREM-1 in HYP exposed neonatal mice led to decreased neutrophil recruitment (Figure 3A) and myeloperoxidase concentrations

(Figure 3B) and reduced lung inflammation as demonstrated by decreased concentrations of proinflammatory cytokines IL-6 and $\mathrm{IL}-1 \beta$ in the lungs (Figures $3 \mathrm{C}$ and $3 \mathrm{D}$ ), as well as improved alveolarization as manifested by decreased chord length and increased radial alveolar count (Figures 3E-3G). Furthermore, these improvements in lung alveolarization were also accompanied by decreased staining of 8-oxo- 2 '-deoxyguanosine in the lung (Figure E3). Notably, we did not detect any changes in lung morphometry and inflammation in the RA groups of neonatal mice treated with TREM-1 agonist or isotype antibodies. Collectively, these results suggest that stimulating TREM-1 activation using the TREM-1 agonist antibody protects against HALI through reduction of lung inflammation.

Previously, TREM-1 has been shown to be crucial for modulating macrophage polarization $(19,20)$. We reasoned that TREM-1 activation may also polarize macrophages to the M2 phenotype to result in a protective response in the lung during HYP. Consistent with this hypothesis, we found that TREM-1 activation polarizes macrophages to the M2 phenotype as manifested by increased concentrations of M2 markers (Ym1, KLF4 , and arginine 1) and decreased expression of M1 markers (inducible nitric oxide synthase, IL-6, and Ccl2) in whole lungs and AM (Figures $3 \mathrm{H}-3 \mathrm{~K}$ ). These data suggest that TREM-1 activation is required to resolve lung inflammation and injury during HYP exposure in the developing lung.

\section{Increased NLRP3 Inflammasome Activation Is Associated with Increased Lung Necroptosis in Human Neonates with RDS and BPD}

Recently, it has been reported that the NLRP3 inflammasome, a key mediator of inflammation, is activated in the lungs of neonatal mice (3) and rats (21) exposed to HYP and in the TA of preterm infants with respiratory failure (3). Consistent with these previous findings, we detected an increased expression of NLRP3 inflammasome markers-NLRP3, caspase 1 , and IL-1 $\beta$-in human neonatal lungs at various stages of BPD development (Figure 4A) and in the lungs of neonatal mice exposed to HYP (Figure E4). These results further strengthen the involvement of NLRP3 inflammasomal activation in the development of BPD; however, the mechanisms for NLRP3 inflammasome activation are not known.

We have previously shown that the HMGB1 (high-mobility group box 1) protein, which is released from necrotic cells, is higher in TA from premature infants who develop BPD (22), and now we have confirmed our earlier result with the increased HMGB1 concentrations in the lungs of human infants developing BPD (Figure 4B). These findings suggest that lung necroptosis may have a crucial role in the development of BPD. Necroptosis, a form of programmed necrotic cell death, amplifies NLRP3 inflammasome activation $(23,24)$ and has been shown to play a critical role in lung diseases such as adult respiratory distress syndrome (ARDS) (4, 25-27), chronic obstructive pulmonary disease (COPD) (28), and sepsis $(29,30)$. We reasoned that increased NLRP3 inflammasome activation might also be functionally related to increased necroptosis in the lungs of human infants developing BPD and neonatal mice exposed to HYP. To test this possibility, we first verified the expression and activation of RIPK3, a regulator protein in the necroptosis pathway in human lungs of neonates with RDS and BPD and in the lungs of neonatal mice exposed to HYP. As shown in Figure 4B, phosphorylation of RIPK3 was significantly increased in human neonatal lungs at various stages of BPD development. Consistent with increased RIPK3 activation in human

Figure 1. (Continued). air- and hyperoxia-exposed neonatal mice at PN7. ( $E$ and $F$ ) A time-course hyperoxia experiment showed increased transcript and protein concentrations of TREM-1 in murine macrophage-like RAW 264.7 cells. (G) Increased TREM-1 mRNA expression in cell pellets obtained from tracheal aspirates in the first postnatal week among neonates who subsequently did or did not develop BPD and/or died. (H) Western blot analysis of TREM-1 expression in human neonatal lungs with no lung disease, mild RDS, evolving BPD, and established BPD. Densitometric analysis is shown to the right. () Immunohistochemical staining for TREM-1 (brown stain) in human neonatal lungs. Increased brown staining (TREM-1 positive) is noted in inflammatory cells in the lungs of neonates with RDS and BPD compared with those of control subjects. Scale bars: $200 \mu \mathrm{m}$ for $\times 100$ and $20 \mu \mathrm{m}$ for $\times 400$. Data are expressed as mean \pm SEM. ${ }^{\star} P<0.05,{ }^{\star \star} P \leqslant 0.01$, and ${ }^{\star \star \star} P \leqslant 0.001$ by Student's unpaired $t$ test and one-way ANOVA followed by Tukey's post hoc analysis. HYP = hyperoxia; RA = room air. 
A

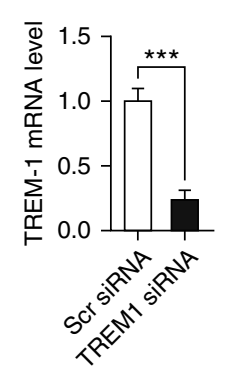

B

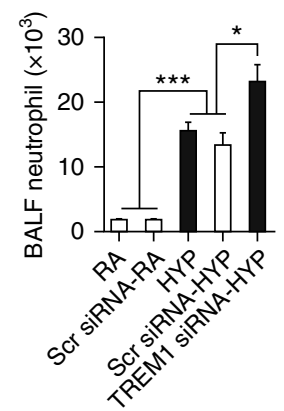

C

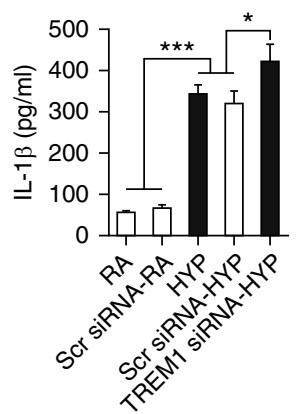

D

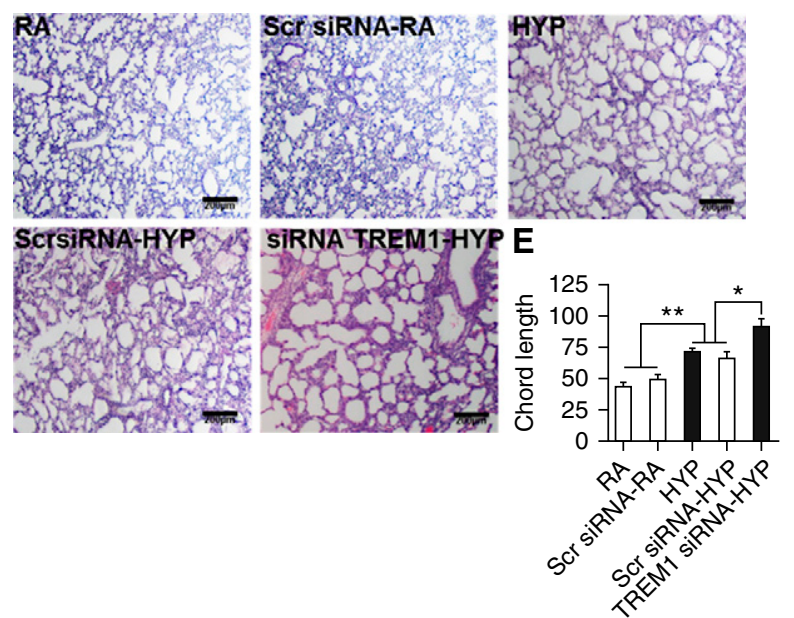

H

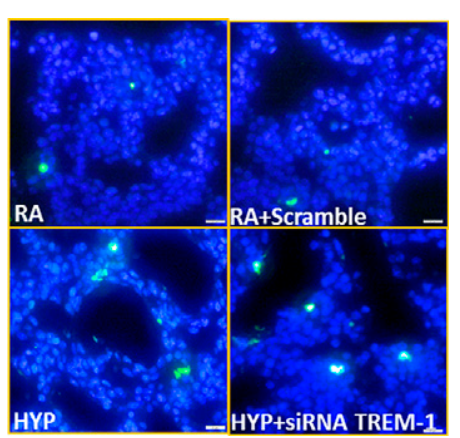

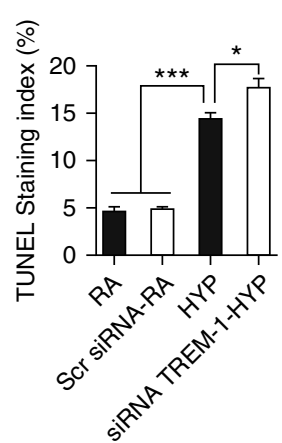

L

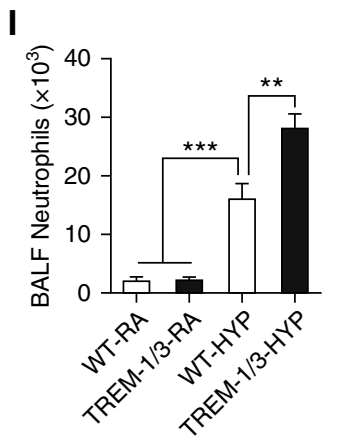

J

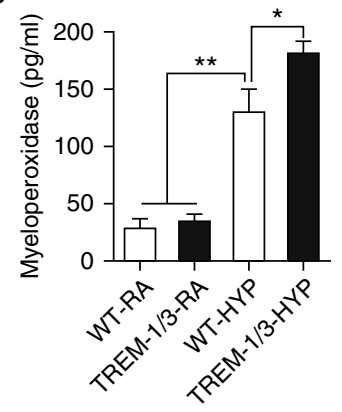

K

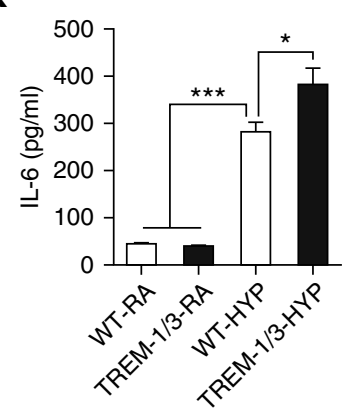

0

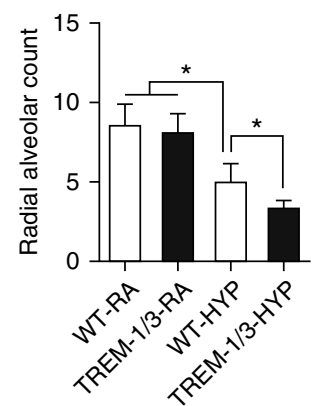

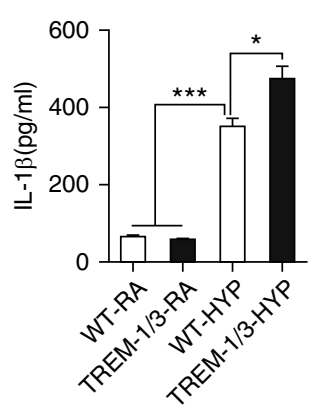

M

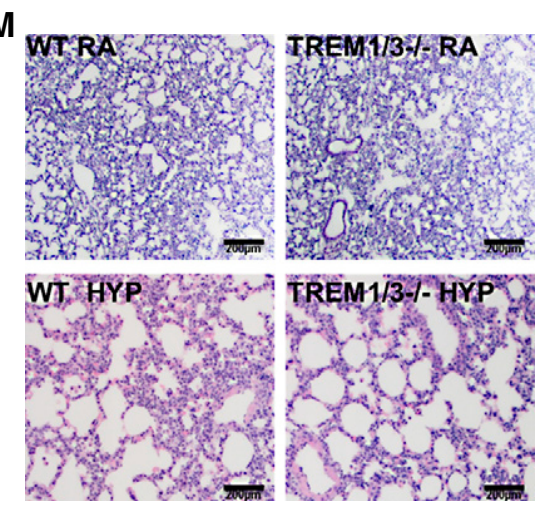

N

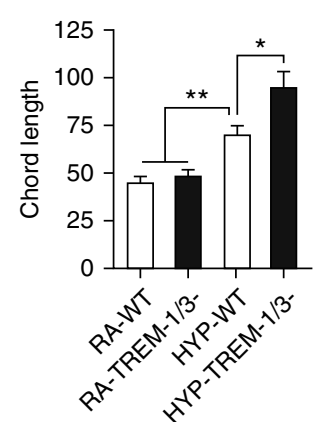

$\mathbf{P}$

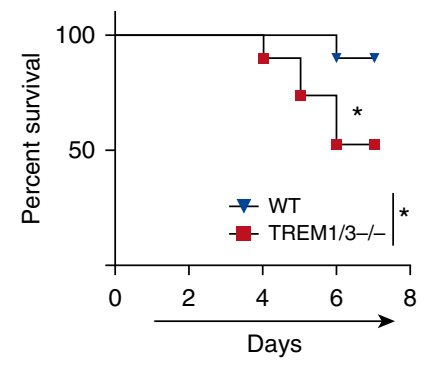

Figure 2. Deletion of TREM-1 is associated with increased pulmonary alveolar injury and mortality of hyperoxia-exposed neonatal mice. (A) TREM-1 siRNA suppresses TREM-1 expression in the lungs of hyperoxia-exposed neonatal mice. (B) Hyperoxia caused a significant increase in neutrophil recruitment to the lungs of newborn wild-type (WT) mice receiving TREM-1 siRNA. (C) TREM-1 siRNA caused increased inflammatory cytokine IL-1 $\beta$ in the lung. $(D-F)$ Chord length and radial alveolar count in lungs of neonatal mice receiving TREM-1 siRNA and scrambled (Scr) siRNA. Measurements 
neonatal lungs at various stages of BPD, neonatal mice exposed to HYP also showed increased activation of RIPK 3 in the lungs (Figure E5).

To further explore the involvement of RIPK3-mediated necroptosis, we measured MLKL (mixed lineage kinase domain-like protein), a downstream substrate of RIPK3 that is necessary for RIPK3 kinase activity to execute necroptosis $(3,23)$. We found increased phosphorylation of MLKL in the lungs of human infants with RDS and BPD (Figure 4B), as well as in the lungs of neonatal mice exposed to HYP (Figure E5). Collectively, these results from human infants with RDS/BPD and HYP-exposed neonatal mice suggest that lung necroptosis during HYP may be involved in the activation of the NLRP3 inflammasome in the neonatal lung.

\section{Inhibition or Deletion of RIPK3 Reduces Necroptosis and NLRP3 Inflammasome Activation}

Next, we examined the functional relationship between RIPK3-mediated necroptosis and NLRP3 inflammasome activation using pharmacological or molecular approaches. We repressed RIPK3 activity using the RIPK3 inhibitor GW440139B (0.5 $\mu \mathrm{M})$ (also known as $\left.\mathrm{GW}^{\prime} 39 \mathrm{~B}\right)$, which disrupts the RIPK3-MKLKL complex and inhibits necroptosis (31), and we also used RIPK3specific null mutant mice to confirm the role of RIPK3-mediated necroptosis in NLRP3 inflammasome activation. We found that RIPK3 inhibitor GW'39B significantly decreased phosphorylation of MLKL, which is required for executing necroptosis (Figure 5A). This decreased lung necroptosis was also associated with diminished NLRP3 inflammasome activation, as manifested by decreased NLRP3, activated caspase 1 , and IL-1 $\beta$ expression in lungs of HYP-exposed neonatal mice (Figure 5B), suggesting that necroptotic cell death during HYP mediates NLRP3 inflammasome activation in the lung. We next examined whether inhibition of RIPK3 also ameliorated pulmonary inflammation and alveolar injury in HYP-exposed neonatal mice. As shown in Figures 5C-5F, RIPK3 inhibition attenuated lung inflammation, as measured by decreased neutrophil recruitment; myeloperoxidase; and concentrations of proinflammatory cytokines TNF- $\alpha$, IL-6, and IL- $1 \beta$ in the lungs. This reduction in lung inflammation was associated with a marked reduction in pulmonary alveolar injury, as manifested by improved alveolarization (decreased chord length and increased radial alveolar count), compared with the HYP group not receiving any treatment (Figures 5G, 5H, and E6).

Similar to the results of RIPK3 inhibitor $\mathrm{GW}^{\prime} 39 \mathrm{~B}$, the RIPK3 null mutant mice also showed robust protection against HYP-induced acute lung inflammation and alveolar injury by reducing lung necroptosis and NLRP3 inflammasome activation (Figures 5I-5R). Altogether, these findings indicate that suppression of RIPK3 expression by an RIPK3 inhibitor or genetic deletion of $R I P K 3$ reduces lung inflammation and attenuates pulmonary alveolar damage in HYP-exposed neonatal mice through inhibition of NLRP3 inflammasome activation.

\section{TREM-1 Deletion Is Associated with Increased Lung Necroptosis and NLRP3 Inflammasome Activation}

Because we found that TREM-1 inhibition induced lung inflammation in HYP-exposed neonatal mice, we reasoned that TREM-1 may be involved in lung necroptosis and NLRP3 inflammasome activation. To test this hypothesis, we examined the markers for RIPK3-mediated necroptosis and NLRP3 inflammasome activation in TREM-1/3-deficient mice exposed to HYP and compared them with WT littermates. As shown in Figure E7A, activated forms of RIPK3 and its downstream substrate phosphorylation of MLKL are increased in the lungs of TREM-1/3-deficient neonatal mice exposed to HYP as compared with
WT neonatal mice. This increased RIPK3-mediated necroptosis is also associated with increased NLRP3 inflammasome activation in TREM-1/3deficient neonatal mice (Figure E7B), suggesting that TREM-1 may be involved in the regulation of RIPK3-mediated lung necroptosis during HYP exposure in neonates.

\section{TREM-1 Activation Suppresses RIPK3-mediated Necroptosis through Induction of Ang1 Expression}

Finally, to address whether diminution of lung inflammation and alveolar injury by TREM-1 activation is arbitrated through the suppression of RIPK3-mediated necroptosis, we treated HYP-exposed neonatal mice with the TREM-1 agonist antibody and measured HMGB1, RIPK3, and its downstream protein MLKL. We found that TREM-1 agonist antibody reduced HMGB1 expression and RIPK3-mediated necroptosis as manifested by decreased expression of RIPK 3 and its downstream protein phosphorylated MLKL in the whole lung as well as in macrophages isolated at PN7 and exposed to HYP in culture (Figures 6A-6D). This decrease in lung necroptosis is also associated with a significant decrease NLRP3 inflammasome activation, as demonstrated by diminished expression of inflammasome markers NLRP3, active caspase 1 , and IL-1 $\beta$ in the lungs of neonatal mice treated with TREM-1 agonist antibody (Figure 6B).

Given the fact that TREM-1 is also found in endothelial cells, we decided to investigate if there was a role for angiogenic agents in this pathway. We found that decreased lung necroptosis was also associated with increased expression of Ang1 and decreased expression of Ang2 proteins (Figures 6C and 6D), suggesting that TREM-1 may show attenuation of necroptosis through activation of Ang1.

To investigate whether Ang1 expression is directly involved in the

Figure 2. (Continued). were taken at $20 \times$ magnification. (G) TREM-1 siRNA caused impaired vascularization. Scale bars: $20 \mu m$. $(H)$ TREM-1 siRNA caused increased cell death in the lungs. Scale bars: $20 \mu \mathrm{m}$. ( / and J) Hyperoxia in TREM-1/3-deficient mice caused increased neutrophil recruitment and myeloperoxidase concentration in the lung. ( $K$ and $L$ ) Hyperoxia in TREM-1/3-deficient mice caused increased inflammatory cytokine IL-6 and IL-1 $\beta$ concentrations in the lung. $(M-O)$ Chord length and radial alveolar count in WT and TREM-1-deficient neonatal mice exposed to hyperoxia for 7 days. Measurements were taken at $\times 10$ magnification. $(P)$ Hyperoxia caused increased mortality in TREM-1/3-deficient neonatal mice. All the data are expressed as mean \pm SEM with $n=10-12$ animals per group. ${ }^{\star} P<0.05$, ${ }^{\star *} P<0.01$, and ${ }^{\star \star \star} P<0.001$ by Student's unpaired $t$ test and one-way ANOVA followed by Tukey's post hoc analysis. BALF = BAL fluid. 
A

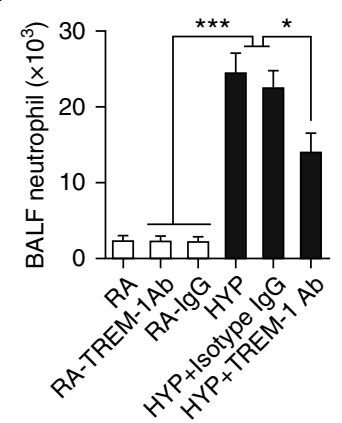

B

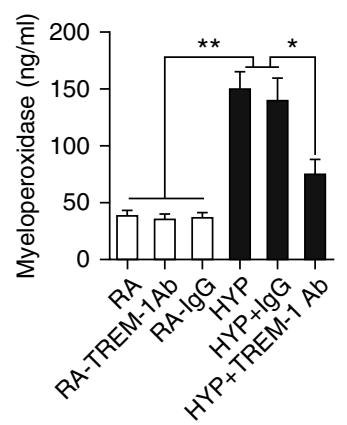

C

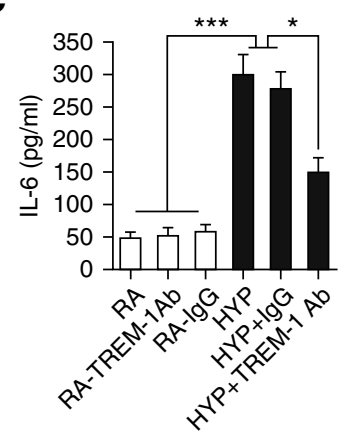

D

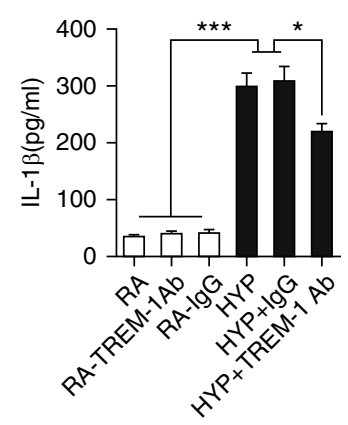

E

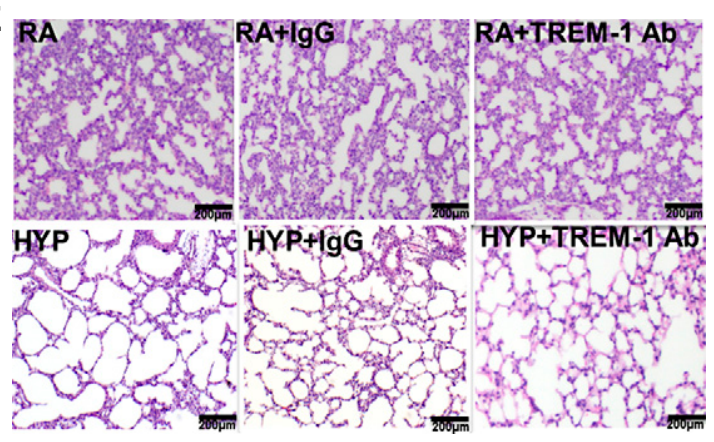

$\mathbf{F}$

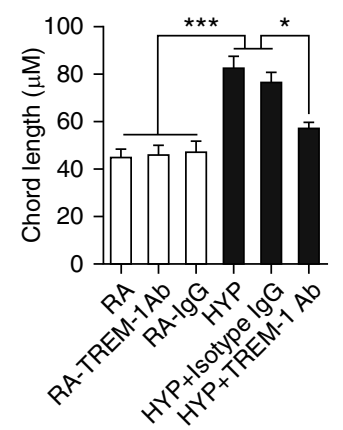

G

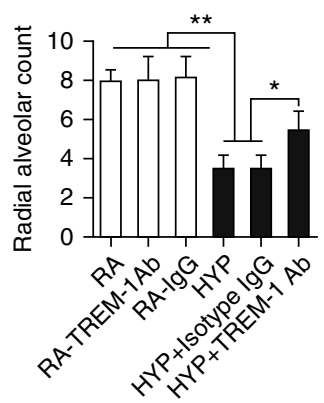

H

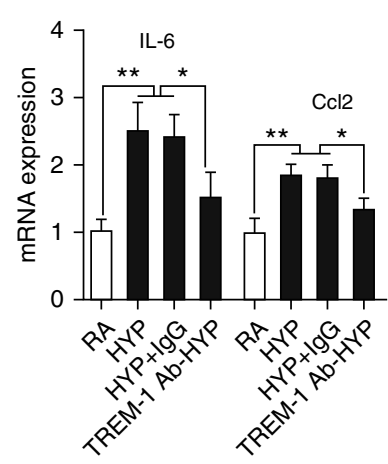

J

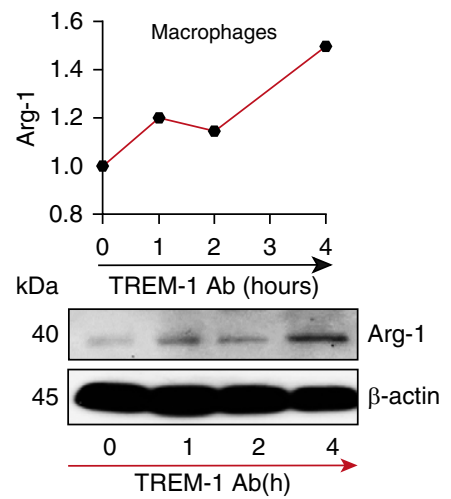

I
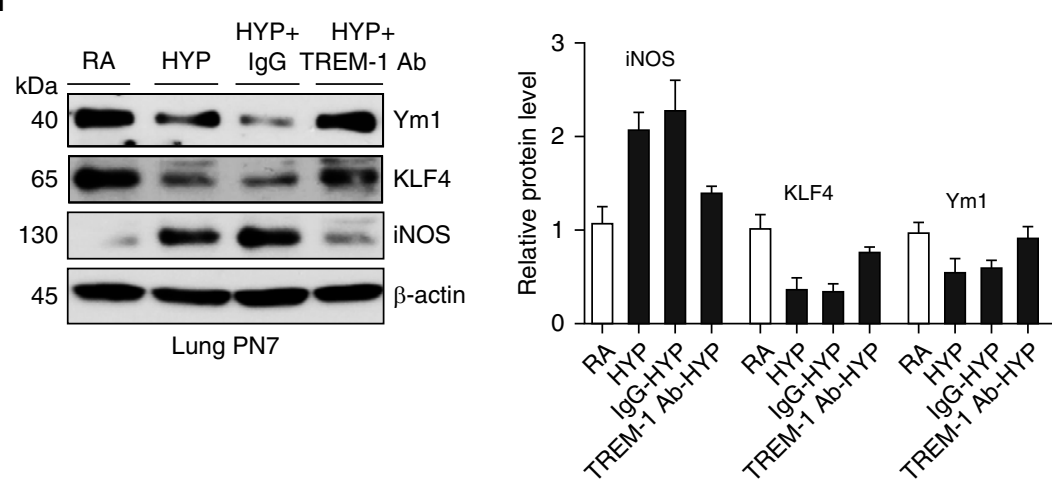

K
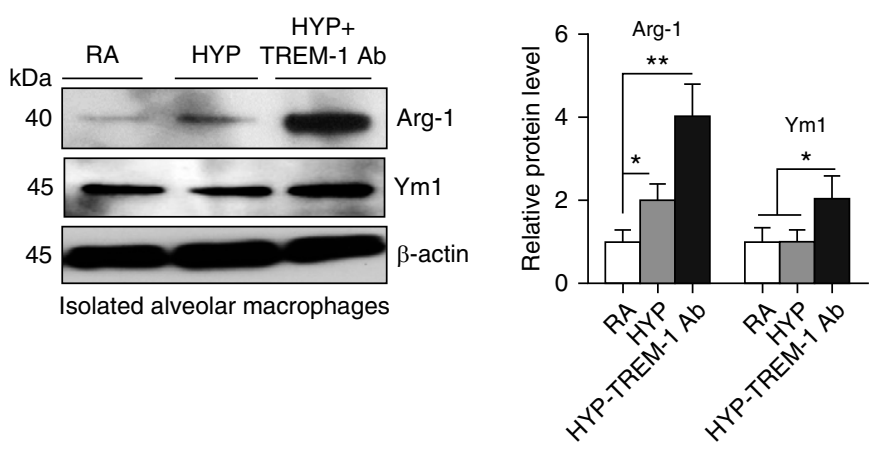

Figure 3. TREM-1 activation decreased pulmonary alveolar injury and inflammation by polarizing macrophages toward the M2 phenotype. (A and $B$ ) Neutrophil recruitment and myeloperoxidase concentrations in the lung significantly decreased in newborn mice treated with TREM-1 agonist antibody (Ab) during HYP. TREM-1 agonist Ab and isotype Ab were given subcutaneously on alternate days starting at PN2. (C and $D$ ) ELISA for IL-6 and IL-1 $\beta$ in lung homogenates from mice treated with TREM-1 agonist $\mathrm{Ab}$ and isotype $\mathrm{Ab}$. ( $E-G)$ Chord length and radial alveolar count in neonatal mice treated with TREM-1 agonist Ab and isotype Ab during HYP exposure. Measurements were taken at $\times 10$ magnification. Scale bars: $200 \mu \mathrm{m}$. 
A
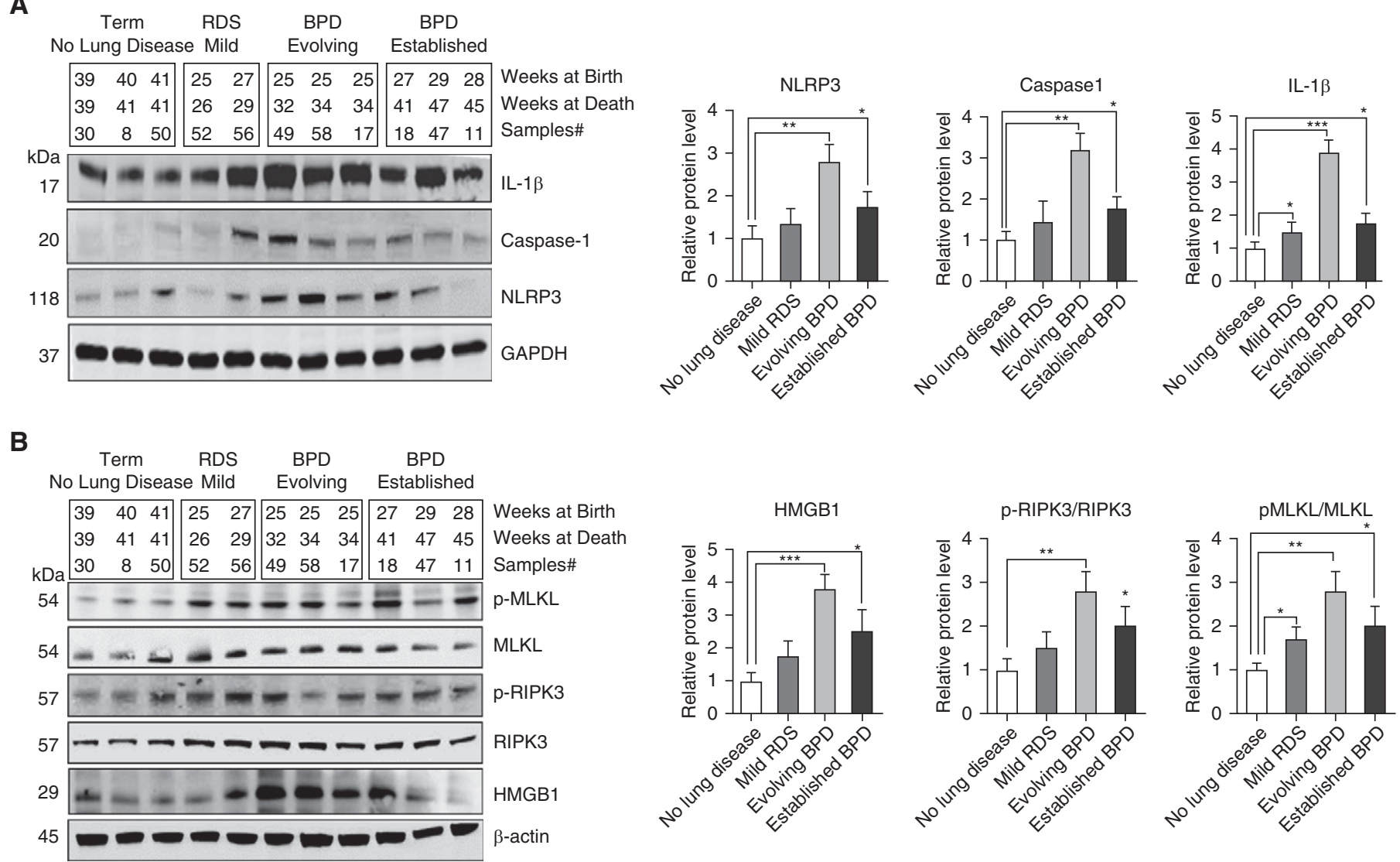

Figure 4. Increased nucleotide-binding oligomerization domain-like receptor containing pyrin domain 3 (NLRP3) inflammasome activation is associated with increased lung necroptosis in human neonates with RDS and BPD. (A) Western blot analysis for NLRP3 inflammasome pathway proteins NLRP3, caspase 1 , and IL-1 $\beta$ in the lungs of human infants with RDS and BPD. Densitometric analyses are shown to the right of the Western blots. (B) Western blot analysis for damage-associated molecular pattern molecules high-mobility group box 1 (HMGB1) and necroptosis pathway proteins receptorinteracting protein kinase 3 (RIPK3) and mixed lineage kinase domain-like (MLKL) protein in the lungs of human infants with RDS and BPD. Densitometric analyses are shown to the right of the Western blots. All the data are expressed as mean \pm SEM. ${ }^{*} P<0.05$, ${ }^{* \star} P<0.01$, and ${ }^{* *} P<0.001$. Statistical significance was assessed using one-way ANOVA followed by Tukey's post hoc analysis. $\mathrm{p}-\mathrm{MLKL}=$ phosphorylated MLKL.

repression of RIPK3 activity, we treated TREM-1/3-deficient mice with recombinant Ang1 during HYP and measured RIPK3-mediated necroptosis and Ang protein expression in the lung. As expected, we found that administration of recombinant Ang1 caused significantly increased Ang1 protein concentrations, enhanced expression of VEGF-A and Tie2 (receptor for Ang1 and Ang2), and decreased concentration of Ang2 in the lungs of TREM-1/3-deficient mice
(Figure 7A). These changes in Ang protein expression were associated with decreased expression of RIPK3 and its downstream substrate MLKL activation in the lungs of TREM-1/3-deficient mice (Figure 7B). We and others have previously shown that increased Ang2, an antagonist of Ang1, causes necrosis upon HYP exposure in developing and adult murine lungs (32, 33). Taken together, our results suggest that TREM-1 may regulate RIPK3mediated necroptosis in part through the
VEGF-A-mediated Ang1/2 signaling pathway in HALI (Figure 7C).

\section{Discussion}

TREM-1 has been reported as an amplifier of the inflammatory response to bacterial infection and sepsis $(7,34,35)$. Several previous studies have shown that treatment with TREM-1 inhibitors can act as a therapeutic strategy to prevent excessive

Figure 3. (Continued). ( $H$ and $I$ TREM-1 augmentation decreased M1 markers (IL-6, inducible nitric oxide synthase [iNOS], Ccl2) and increased concentrations of M2 markers (Ym1 and KLF-4) in the lungs of HYP-exposed newborn mice. ( $)$ Macrophages treated with TREM-1 agonist Ab polarized macrophages to the M2 phenotype in a time-dependent manner. (K) Alveolar macrophages (AM) exposed to HYP and treated with TREM-1 agonist Ab polarized macrophages to the M2 phenotype. All the data are expressed as mean \pm SEM with $n=10-12$ animals per group. For AM experiments, AM were pooled together from three mice and repeated twice. ${ }^{\star} P<0.05$, ${ }^{\star \star} P<0.01$, and ${ }^{\star \star \star} P<0.001$ by Student's unpaired $t$ test and one-way ANOVA followed by Tukey's post hoc analysis. Arg-1 = arginine 1. 
A

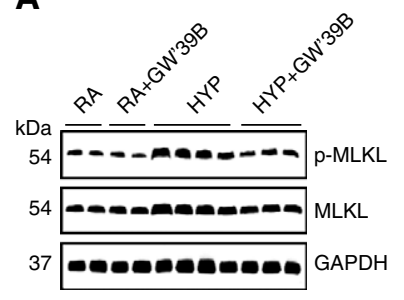

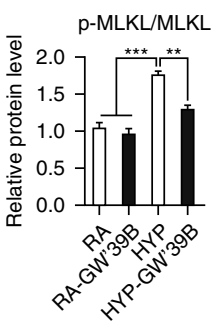

B

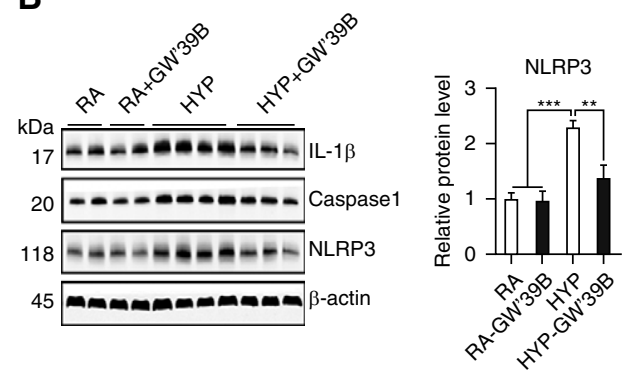

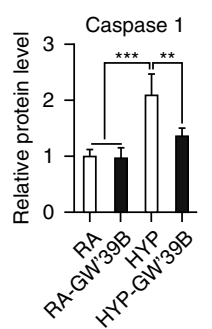

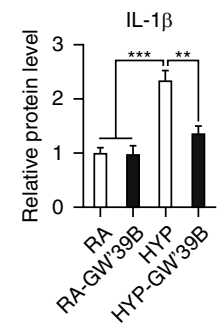

C

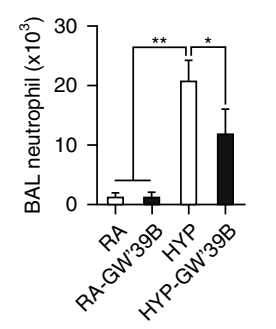

I

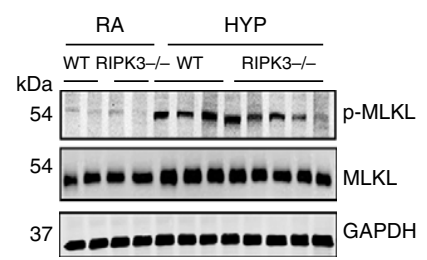

E

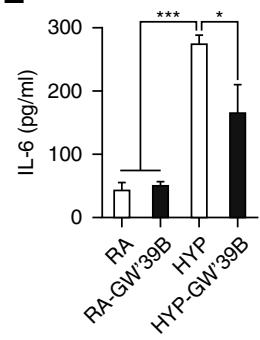

F

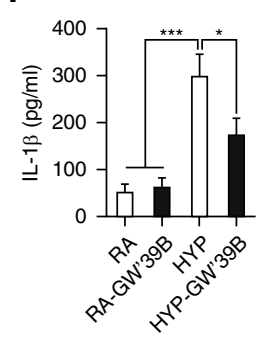

G

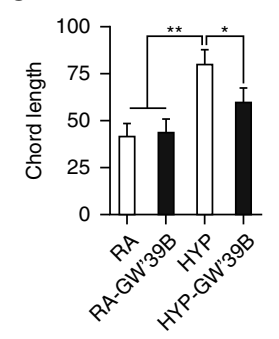

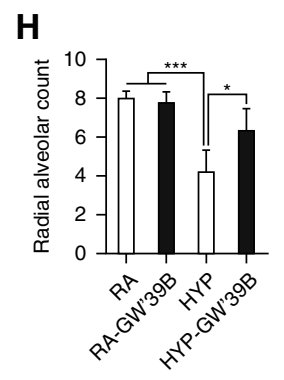

J
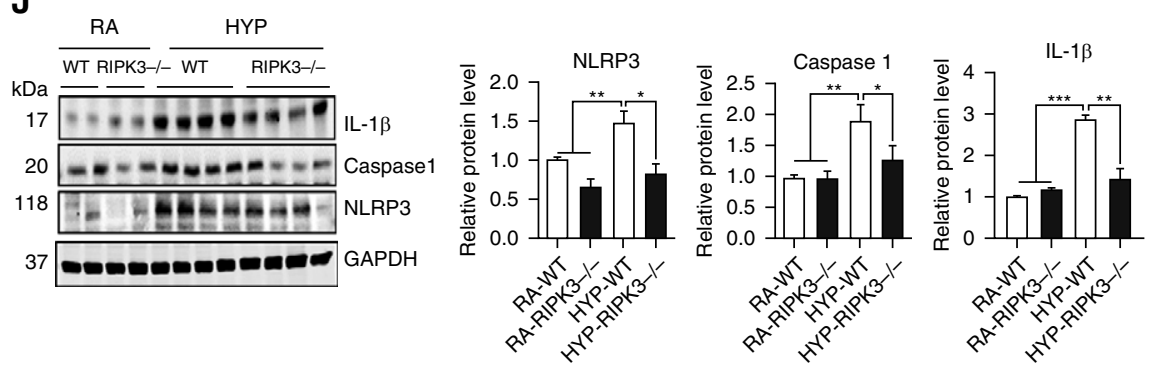

K

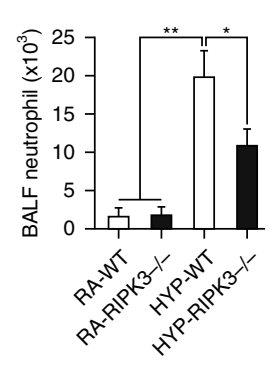

$\mathbf{L}$

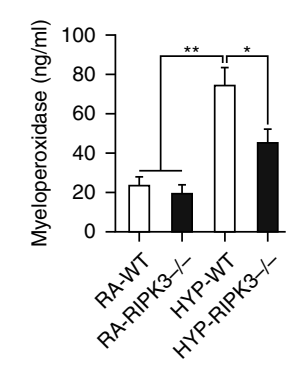

M

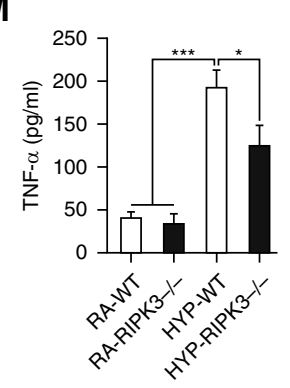

N

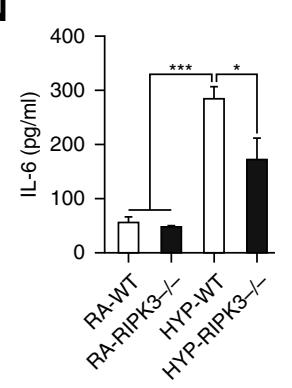

0

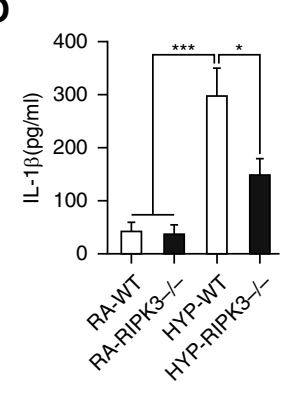

$\mathbf{P}$

Q

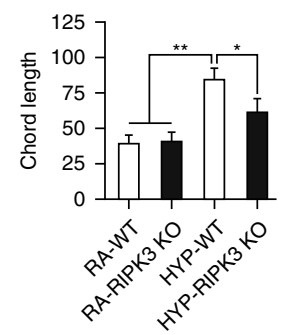

R

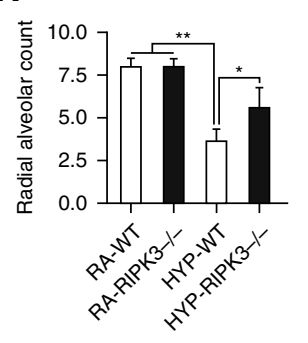

Figure 5. Pharmacological inhibition of RIPK3 and deletion of the RIPK3 gene reduced NLRP3 inflammasome and pulmonary alveolar injury through downregulating necroptosis in HYP-exposed neonatal mice. (A) Western blot analysis for necroptosis-regulating protein RIPK3 and its downstream protein MLKL in the lungs of neonatal mice treated with RIPK3 inhibitor GW440139B (GW'39B) during HYP. Densitometric analysis is shown to the right of the 
systemic inflammation, resulting in decreased severity of sepsis $(34,35)$ and mortality $(19,36)$. In contrast, other studies have recognized TREM-1 as a survival factor for the host against bacterial infection and sepsis (8). For example, it has been demonstrated that deletion of the TREM-1/3 genes in mice leads to increased local as well as systemic cytokine production and enhanced mortality during polymicrobially induced sepsis $(36,37)$. These results suggest that TREM-1 has a sundry role in inflammation and infection, and further investigation is required to explore the functional role of TREM-1 in various diseases. In the present study, we explored a new role of TREM-1 in HYP-induced pulmonary alveolar injury and its consequence in the development of BPD. We demonstrated, for the first time, to our knowledge, that TREM-1 expression is increased in the lungs of neonatal mice and human neonates with RDS and BPD in HYP as an adaptive response to protect the lung from oxygen- and/or ventilationinduced injury. This is evident based on our finding that silencing TREM-1 expression in lungs or genetic deletion of TREM-1 in mice led to increased pulmonary alveolar injury and mortality after exposure to HYP. Furthermore, we observed that augmentation of TREM-1 expression in neonatal murine lungs reduced inflammation and pulmonary alveolar injury and was associated with decreased NLRP3 inflammasome activation and RIPK3-mediated necroptosis.

Previously, it was reported that TREM-1/3-deficient adult mice infected with Pseudomonas aeruginosa showed a markedly increased mortality; however, decreased neutrophil recruitment to the lungs after $P$. aeruginosa challenge was found (8). We report that in the present study, TREM-1/3-deficient neonatal mice have increased neutrophil recruitment during HYP. This discrepancy in results may be due to different stages of lung development/ages of mice (neonate vs. adult) and/or mode of injury (HYP vs. bacterial infection) that we used in our study.

There are certain limitations to our studies. We used an immortalized macrophage cell line derived from the peritoneum of $\mathrm{BALB} / \mathrm{c}$ mice for some of our studies, which may not completely reflect the monocyte/macrophage response in the pulmonary compartment. In addition, the number of clinical samples was quite small, with only three controls.

Whether macrophage or endothelial TREM-1 is more important would be difficult to answer without conducting studies with cell-specific knockouts, which was beyond the scope of the present study. However, targeted deletion of endothelial TREM-1 did protect in an adult sepsis (cecal ligation and puncture) model (38). Ang1 and Ang2 are known to be produced by airway and lung epithelial cells $(33,39)$ The Tie2 receptor (receptor for Ang1 and Ang2) was present in a subset of human and murine circulating monocytes/ macrophages in a previous study (40). The expression of multiple chemokines and cytokines was enhanced in the presence of Ang1 and Ang2 via Tie2 activation of JAK/STAT (Janus kinase/signal transducer and activator of transcription) signaling. The authors of that study concluded that a general role for Tie2 exists in cooperatively promoting the inflammatory activation of macrophages, independently of the polarization state (40). In another study, Ang1 was found to functionally bind to and stimulate monocytes via p38 and Erk1/2 (extracellular signal-regulated kinases 1/2) phosphorylation. Angl switched macrophage differentiation toward a proinflammatory phenotype, even in the presence of an antiinflammatory mediator. These findings suggest that Ang1 plays a role in stimulating proinflammatory responses (41). We observed minimal colocalization of TREM-1 with endothelium in neonatal mouse lungs (Figure E1C). It is important to point out that cell-specific roles of TREM-1 need to be tested in a developmentally appropriate disease context and should not be extrapolated from in vitro or adult in vivo studies.

RIPK3-mediated necroptosis has been identified in several lung diseases, such as ARDS (4, 25-27), COPD (28), and sepsis $(29,30)$. We now add neonatal HALI and BPD to this list and identify TREM-1, Ang1, and RIPK3 as potential therapeutic targets for the treatment of these lifethreatening disorders. In the present study, we reveal that necroptosis-regulating protein RIPK3 and its downstream substrate MLKL concentrations were higher in the lungs of human infants with RDS, evolving BPD, and established BPD, as well as in lungs of neonatal mice exposed to HYP, suggesting the essential role of necroptosis in HALI and the development of BPD. Furthermore, using both genetic and drug-based approaches, we revealed a decisive role of RIPK3 in HALI in neonatal mice. Our mechanistic data showed that RIPK3 inhibitor GW'39B- or RIPK3deficient neonatal mice exposed to HYP showed decreased phosphorylation of MLKL and diminished necroptosis and NLRP3 inflammasome activation in the lung. These findings are similar to those of the recent studies of Wang and colleagues (27) and Mizumura and colleagues (28), who showed that RIPK3-mediated necroptosis is a major mechanism for lung inflammation and injury in experimental models of ARDS and COPD. It is important to point out that although individual cytokine concentrations may not dramatically differ (even though the differences were statistically significant), in the context of the pulmonary compartment of a developing lung, and in concert with other inflammatory mediators, we believe that they would potentially be clinically

Figure 5. (Continued). Western blots. (B) Western blot analysis for NLRP3 inflammasome pathway proteins NLRP3, caspase 1 , and IL-1 $\beta$ in the lungs of neonatal mice treated with RIPK3 inhibitor GW'39B during HYP. Densitometric analysis is shown below each Western blot. (C and $D)$ Neutrophil cell counts in the BALF and myeloperoxidase concentrations in the lungs of neonatal mice treated with RIPK3 inhibitor GW'39B during HYP. (E and $F$ ) IL-6 and IL-1 $\beta$ measured by ELISA in the lungs of neonatal mice treated with RIPK3 inhibitor GW'39B during HYP. ( $G$ and $H$ ) Chord length and radial alveolar count in the lungs of neonatal mice treated with RIPK3 inhibitor GW'39B during HYP. (I-R) RIPK3 ${ }^{-1-}$ mice are protected against HYP-induced lung inflammation and alveolar damage in the lungs. Measurements were taken at 10x magnification. All the data are expressed as mean \pm SEM with $n=10-12$ animals per group. ${ }^{\star} P<0.05,{ }^{\star *} P<0.01$, and ${ }^{* \star} P<0.001$. Statistical significance was assessed using one-way ANOVA followed by Tukey's post hoc analysis. Scale bars: $200 \mu \mathrm{m}$. $\mathrm{KO}=$ knockout. 

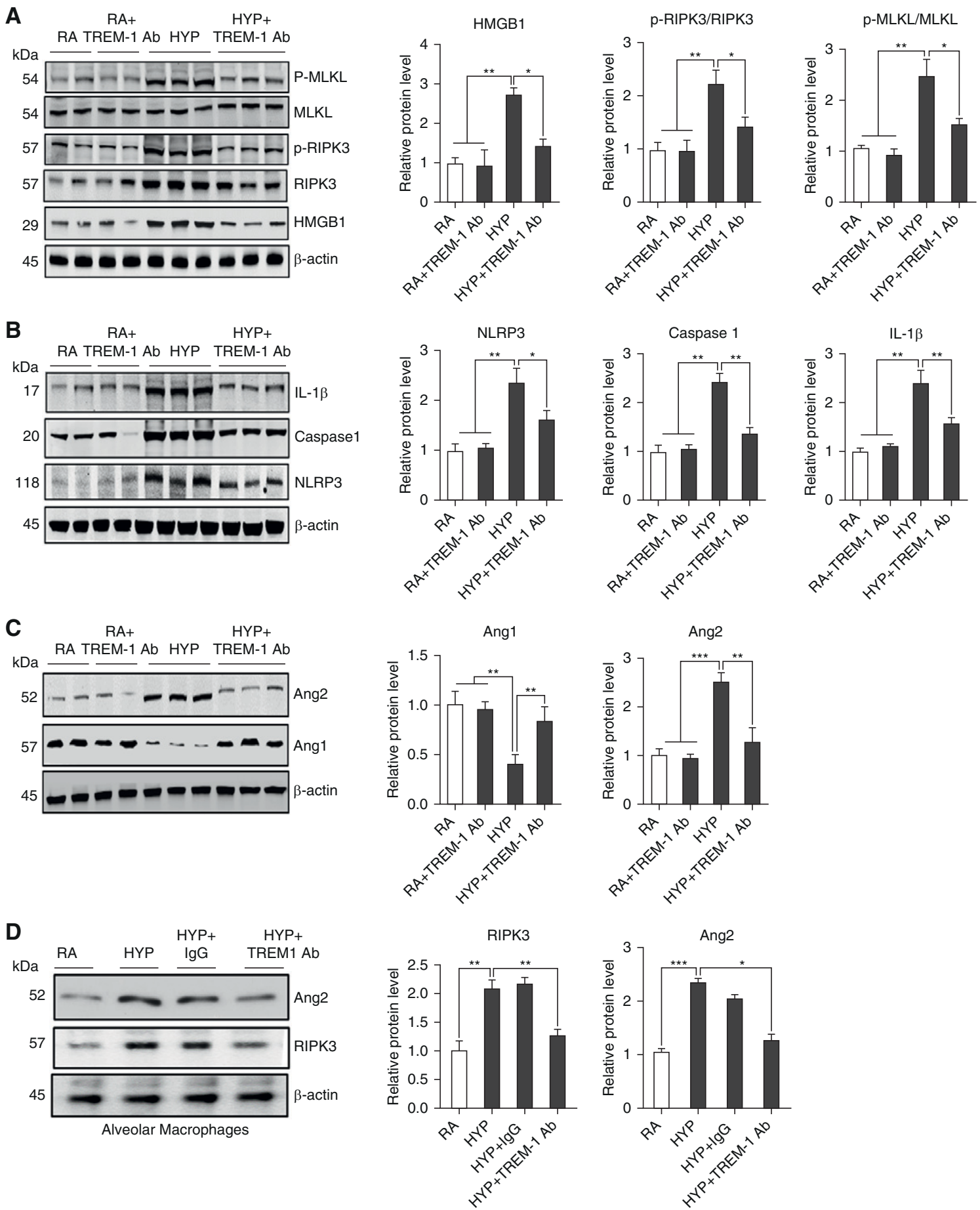

Figure 6. TREM-1 activation suppressed RIPK3-mediated necroptosis and NLRP3 inflammasome in lungs. (A) Western blot analysis for damageassociated molecular pattern molecule HMGB1 and necroptosis pathway proteins MLKL and p-MLKL in the lungs of neonatal mice treated with TREM-1 agonist Ab during HYP exposure. Densitometric analysis is shown to the right of the immunoblots. (B) Western blot analysis for NLRP3 inflammasome proteins NLRP3, caspase 1 , and IL-1 $\beta$ in the lungs of neonatal mice treated with TREM-1 agonist Ab during HYP exposure.

Densitometric analysis is shown to the right of the immunoblots. (C) Western blot analysis for angiopoietins 1 and 2 (Ang1 and Ang2, respectively) in the lungs of wild-type neonatal mice treated with TREM-1 agonist Ab during HYP. Densitometric analysis is shown to the right of the immunoblots. (D) Western blot analysis for Ang2 and RIPK3 in the alveolar macrophage culture with TREM-1 agonist Ab and exposed to HYP for 16 hours. Densitometric analysis is shown on the right. All the data are expressed as mean \pm SEM with $n=6-8$ animals per group. For AM experiments, AMs were pooled together from three mice and repeated twice. ${ }^{\star} P<0.05$, ${ }^{\star \star} P<0.01$, and ${ }^{\star \star \star} P<0.001$. Statistical significance was assessed using Student's unpaired $t$ test and one-way ANOVA followed by Tukey's post hoc analysis. 
A

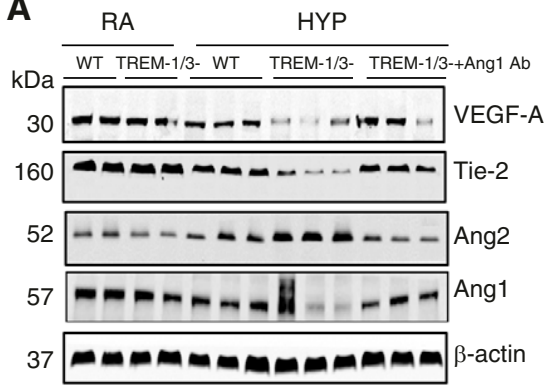

B

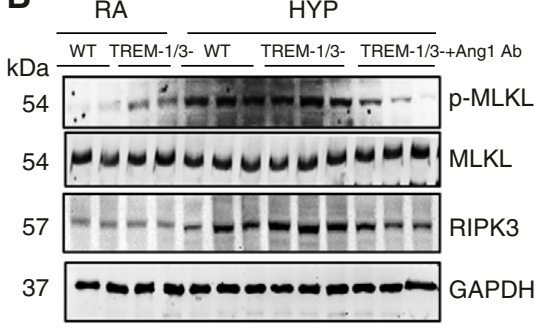

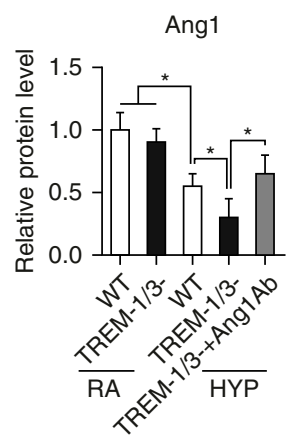
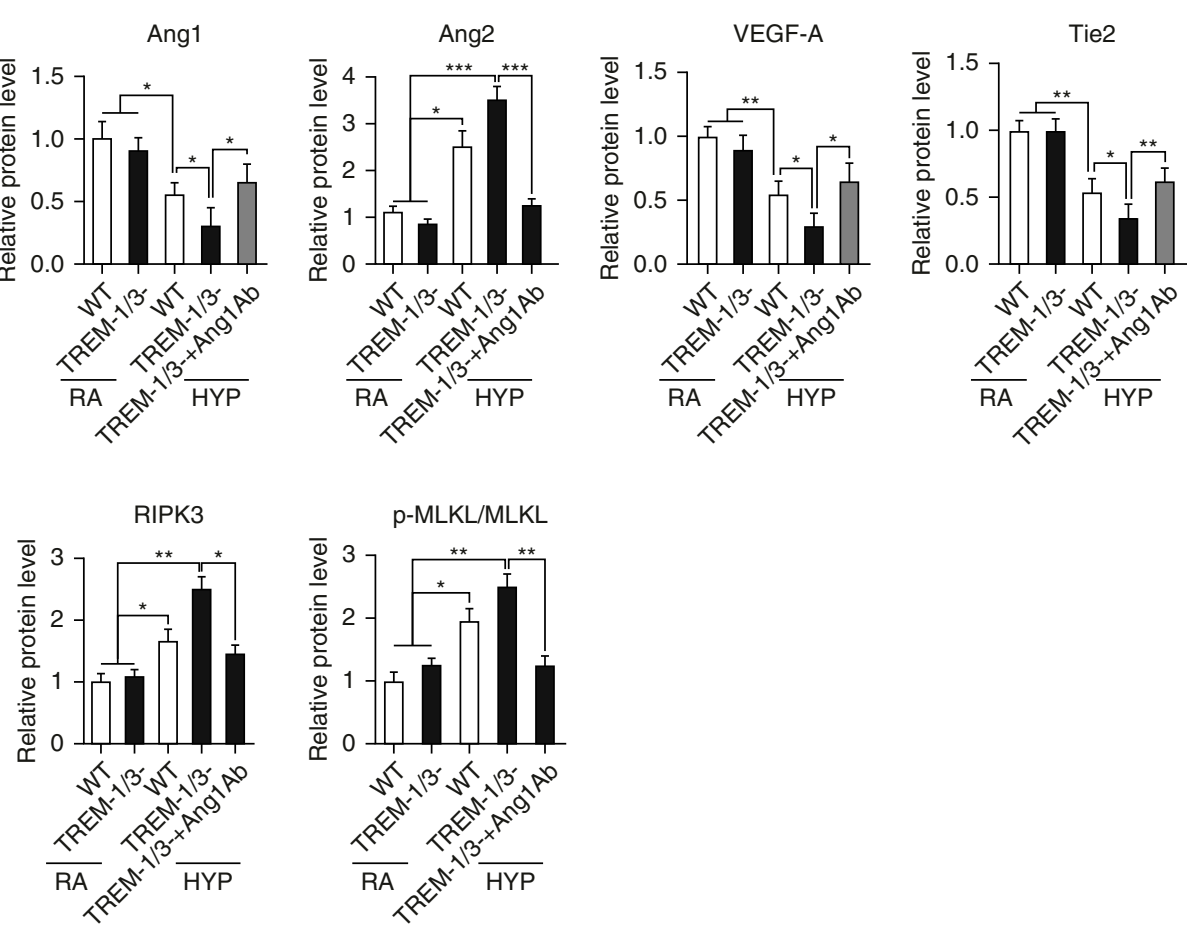

C

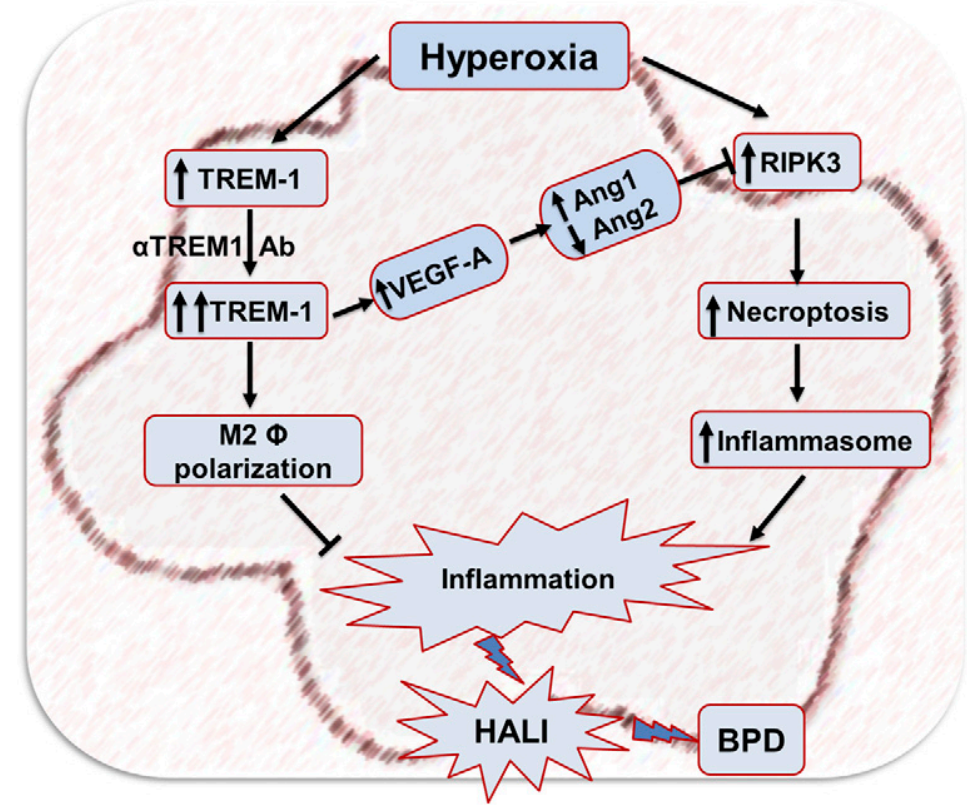

Figure 7. TREM-1 activation suppressed RIPK3-mediated necroptosis through induction of angiopoietin 1 (Ang1) expression in lungs. (A and $B$ ) Western blot analysis for Ang1, Ang2, RIPK3, and p-MLKL in the lungs of WT and TREM-1/3-deficient neonatal mice treated with recombinant Ang1 during HYP exposure. Densitometric analysis is shown to the right of the immunoblots. $(C)$ Schematic illustration of the proposed sequence of events leading to hyperoxia-induced acute lung injury (HALI) and BPD after HYP exposure to developing lungs. TREM-1 alleviates pulmonary alveolar injury and inflammation through downregulating necroptosis protein RIPK3 and NLRP3 inflammasome activation in HYP-exposed developing lungs. All the data are expressed as mean \pm SEM with $n=6-8$ animals per group. ${ }^{*} P<0.05$, ${ }^{\star \star} P<0.01$, and ${ }^{\star * *} P<0.001$. Statistical significance was assessed using Student's unpaired $t$ test and one-way ANOVA followed by Tukey's post hoc analysis. Tie-2 = receptor for Ang1 and Ang2; VEGF = vascular endothelial growth factor. 
relevant. Although our study is primarily focused on TREM1-mediated lung necroptosis in HALI, our group has previously shown TREM-1 as an antiapoptotic protein that prolongs macrophage survival by inducing Bcl-2 in an Egr2 (early growth response 2)dependent manner in response to LPS exposure (19).

Another important finding of this study is that we illustrated, for the first time, to our knowledge, that the inflammation mediator NLRP3 inflammasome is regulated by RIPK3-mediated necroptosis in HALI in neonatal mice. Recently, the NLRP3 inflammasome has been shown to be activated in neonatal mice exposed to HYP $\left(85 \% \mathrm{O}_{2}\right)$ and in a preterm baboon model of BPD (3). The blocking of NLRP3 inflammasome resulted in decreased lung inflammation and improved pulmonary alveolarization; however, the mechanism for NLRP3 inflammasome activation during HYP has not been explored. We showed that NLRP3 inflammasome activation may be due to increased necroptosis during HYP in neonatal mice. This is supported by our finding that RIPK3 inhibitor GW'39B and genetic deletion of RIPK3 in mice are related to decreased NLRP3 inflammasome activation, caspase 1 , and IL- $1 \beta$ in neonatal mice exposed to HYP. These results are further supported by several earlier studies showing that multiple damage-associated molecular patterns (HMGB1, histones, and mitochondrial DNA) released by necroptotic cells triggered sustained cytokine release and amplified the inflammatory response in diseases $(22,42-44)$. Consistent with this line of evidence, we have also previously shown increased concentrations of HMGB1 in the TA of infants who developed BPD (22), and now we show that there is an increase in HMGB1 and caspase 8 expression in the lungs of human infants with BPD as well as in neonatal mice exposed to HYP. Collectively, these data suggest that the activation of the NLRP3 inflammasome may be due to increased necroptosis in the lungs of infants exposed to invasive ventilation/HYP, which leads to the development of BPD.

Last, we demonstrated that the protection conferred by augmenting TREM-1 expression strongly suppressed the activity of RIPK3 and decreased the phosphorylation of its downstream substrate MLKL, which is required to execute necroptosis $(45,46)$. Although TREM-1 has been known to be a pro- as well as antiinflammatory molecule that has contributed to its ability to exacerbate or mitigate inflammation in the lung, respectively, we explored a new function of TREM-1 that confers protection in HYPexposed neonatal mice through induction of Ang1 and suppression of Ang2 in neonatal mice treated with TREM-1 agonist antibody.

We have previously reported that Ang2 increases inflammation and necroptotic cell death during HYP in mice $(32,33)$. These findings, together with the results of the present study, indicate that TREM-1 is a functionally important protein in the neonatal lung for improved alveolarization and survival in HYP. In the future, it will be important to clarify the precise mechanism by which TREM-1 regulates Ang1/Ang2 signaling in HALI and BPD in neonatal mice.

In summary, our results suggest that RIPK3-dependent necrosis is the common denominator between TREM-1 signaling and development of BPD as a consequence of HALI in neonatal mice. We conclude that TREM-1 functions by attenuating necroptosis and NLRP3 inflammation in neonatal murine lungs exposed to HYP, and we identify TREM-1, Ang1, and RIPK3 as potential therapeutic targets for the treatment of HALI and BPD in human preterm newborns.

Author disclosures are available with the text of this article at www.atsjournals.org.

\section{References}

1. Bhandari V. Hyperoxia-derived lung damage in preterm infants. Semin Fetal Neonatal Med 2010;15:223-229.

2. Harijith AK, Bhandari V. Hyperoxia in the pathogenesis of bronchopulmonary dysplasia. In: Bhandari V, editor. Bronchopulmonary dysplasia. Cham, Switzerland: Humana Press; 2016. pp. 3-26.

3. Liao J, Kapadia VS, Brown LS, Cheong N, Longoria C, Mija D, et al. The NLRP3 inflammasome is critically involved in the development of bronchopulmonary dysplasia. Nat Commun 2015;6:8977.

4. Jones HD, Crother TR, Gonzalez-Villalobos RA, Jupelli M, Chen S, Dagvadorj J, et al. The NLRP3 inflammasome is required for the development of hypoxemia in LPS/mechanical ventilation acute lung injury. Am J Respir Cell Mol Biol 2014;50:270-280.

5. Tolle LB, Standiford TJ. Danger-associated molecular patterns (DAMPs) in acute lung injury. $J$ Pathol 2013;229:145-156.

6. Colonna M. TREMs in the immune system and beyond. Nat Rev Immunol 2003;3:445-453.

7. Sharif $\mathrm{O}$, Knapp S. From expression to signaling: roles of TREM-1 and TREM-2 in innate immunity and bacterial infection. Immunobiology 2008;213:701-713.

8. Klesney-Tait J, Keck K, Li X, Gilfillan S, Otero K, Baruah S, et al. Transepithelial migration of neutrophils into the lung requires TREM-1. J Clin Invest 2013;123:138-149.

9. Newton K, Sun X, Dixit VM. Kinase RIP3 is dispensable for normal NF$\kappa \mathrm{Bs}$, signaling by the B-cell and T-cell receptors, tumor necrosis factor receptor 1, and Toll-like receptors 2 and 4. Mol Cell Biol 2004; 24:1464-1469.
10. Syed M, Das P, Pawar A, Aghai ZH, Kaskinen A, Zhuang

ZW, et al. Hyperoxia causes miR-34a-mediated injury via angiopoietin-1 in neonatal lungs. Nat Commun 2017;8: 1173.

11. Sureshbabu A, Syed M, Das $P$, Janér $C$, Pryhuber G, Rahman A, et al. Inhibition of regulatory-associated protein of mechanistic target of rapamycin prevents hyperoxia-induced lung injury by enhancing autophagy and reducing apoptosis in neonatal mice. Am J Respir Cell Mol Biol 2016;55:722-735.

12. Shah D, Romero F, Stafstrom W, Duong M, Summer R. Extracellular ATP mediates the late phase of neutrophil recruitment to the lung in murine models of acute lung injury. Am J Physiol Lung Cell Mol Physiol 2014;306:L152-L161.

13. Shah D, Romero F, Zhu Y, Duong M, Sun J, Walsh K, et al. C1q deficiency promotes pulmonary vascular inflammation and enhances the susceptibility of the lung endothelium to injury. J Biol Chem 2015; 290:29642-29651.

14. Sun $\mathrm{H}$, Choo-Wing R, Sureshbabu A, Fan J, Leng L, Yu S, et al. A critical regulatory role for macrophage migration inhibitory factor in hyperoxia-induced injury in the developing murine lung. PLoS One 2013;8:e60560.

15. Bhandari V, Choo-Wing R, Harijith A, Sun H, Syed MA, Homer RJ, et al Increased hyperoxia-induced lung injury in nitric oxide synthase 2 null mice is mediated via angiopoietin 2. Am J Respir Cell Mol Biol 2012;46:668-676.

16. Berger J, Bhandari V. Animal models of bronchopulmonary dysplasia: the term mouse models. Am J Physiol Lung Cell Mol Physiol 2014; 307:L936-L947. 
17. Lagler H, Sharif $\mathrm{O}$, Haslinger I, Matt U, Stich K, Furtner T, et al. TREM-1 activation alters the dynamics of pulmonary IRAK-M expression in vivo and improves host defense during pneumococcal pneumonia. J Immunol 2009;183:2027-2036.

18. Yang C, Chen B, Zhao J, Lin L, Han L, Pan S, et al. TREM-1 signaling promotes host defense during the early stage of infection with highly pathogenic Streptococcus suis. Infect Immun 2015;83:3293-3301.

19. Yuan Z, Syed MA, Panchal D, Joo M, Colonna M, Brantly M, et al. Triggering receptor expressed on myeloid cells 1 (TREM-1)-mediated Bcl-2 induction prolongs macrophage survival. J Biol Chem 2014; 289:15118-15129.

20. Chen LC, Laskin JD, Gordon MK, Laskin DL. Regulation of TREM expression in hepatic macrophages and endothelial cells during acute endotoxemia. Exp Mol Pathol 2008;84:145-155.

21. Hummler JK, Dapaah-Siakwan F, Vaidya R, Zambrano R, Luo S, Chen $\mathrm{S}$, et al. Inhibition of Rac1 signaling downregulates inflammasome activation and attenuates lung injury in neonatal rats exposed to hyperoxia. Neonatology 2017;111:280-288.

22. Aghai ZH, Saslow JG, Meniru C, Porter C, Eydelman R, Bhat V, et al. High-mobility group box-1 protein in tracheal aspirates from premature infants: relationship with bronchopulmonary dysplasia and steroid therapy. J Perinatol 2010;30:610-615.

23. Lawlor KE, Khan N, Mildenhall A, Gerlic M, Croker BA, D'Cruz AA, et al. RIPK3 promotes cell death and NLRP3 inflammasome activation in the absence of MLKL. Nat Commun 2015;6:6282.

24. Kang TB, Yang SH, Toth B, Kovalenko A, Wallach D. Caspase-8 blocks kinase RIPK3-mediated activation of the NLRP3 inflammasome. Immunity 2013;38:27-40.

25. Grailer JJ, Canning BA, Kalbitz M, Haggadone MD, Dhond RM, Andjelkovic AV, et al. Critical role for the NLRP3 inflammasome during acute lung injury. $J$ Immunol 2014;192:5974-5983.

26. Pan L, Yao DC, Yu YZ, Li SJ, Chen BJ, Hu GH, et al. Necrostatin-1 protects against oleic acid-induced acute respiratory distress syndrome in rats. Biochem Biophys Res Commun 2016;478: 1602-1608.

27. Wang L, Wang T, Li H, Liu Q, Zhang Z, Xie W, et al. Receptor interacting protein 3-mediated necroptosis promotes lipopolysaccharideinduced inflammation and acute respiratory distress syndrome in mice. PLoS One 2016;11:e0155723.

28. Mizumura K, Cloonan SM, Nakahira K, Bhashyam AR, Cervo M, Kitada $\mathrm{T}$, et al. Mitophagy-dependent necroptosis contributes to the pathogenesis of COPD. J Clin Invest 2014;124:3987-4003.

29. Mizumura K, Maruoka S, Gon Y, Choi AM, Hashimoto S. The role of necroptosis in pulmonary diseases. Respir Investig 2016;54: 407-412.

30. Duprez L, Takahashi N, Van Hauwermeiren F, Vandendriessche B, Goossens V, Vanden Berghe T, et al. RIP kinase-dependent necrosis drives lethal systemic inflammatory response syndrome. Immunity 2011;35:908-918.
31. González-Juarbe N, Gilley RP, Hinojosa CA, Bradley KM, Kamei A, Gao $\mathrm{G}$, et al. Pore-forming toxins induce macrophage necroptosis during acute bacterial pneumonia. PLoS Pathog 2015;11:e1005337.

32. Bhandari V, Elias JA. The role of angiopoietin 2 in hyperoxia-induced acute lung injury. Cell Cycle 2007;6:1049-1052.

33. Bhandari V, Choo-Wing R, Lee CG, Zhu Z, Nedrelow JH, Chupp GL, et al. Hyperoxia causes angiopoietin 2-mediated acute lung injury and necrotic cell death. Nat Med 2006;12:1286-1293.

34. Bouchon A, Facchetti F, Weigand MA, Colonna M. TREM-1 amplifies inflammation and is a crucial mediator of septic shock. Nature 2001 410:1103-1107.

35. Nathan C, Ding A. TREM-1: a new regulator of innate immunity in sepsis syndrome. Nat Med 2001;7:530-532.

36. Palazzo SJ, Simpson T, Schnapp LM. Triggering receptor expressed on myeloid cells type 1 as a potential therapeutic target in sepsis. Dimens Crit Care Nurs 2012;31:1-6.

37. Liu T, Zhou Y, Li P, Duan JX, Liu YP, Sun GY, et al. Blocking triggering receptor expressed on myeloid cells-1 attenuates lipopolysaccharideinduced acute lung injury via inhibiting NLRP3 inflammasome activation. Sci Rep 2016;6:39473.

38. Jolly L, Carrasco K, Derive M, Lemarié J, Boufenzer A, Gibot S. Targeted endothelial gene deletion of triggering receptor expressed on myeloid cells-1 protects mice during septic shock. Cardiovasc Res 2018;114:907-918.

39. Grzenda A, Shannon J, Fisher J, Arkovitz MS. Timing and expression of the angiopoietin-1-Tie-2 pathway in murine lung development and congenital diaphragmatic hernia. Dis Model Mech 2013;6:106-114.

40. García S, Krausz S, Ambarus CA, Fernández BM, Hartkamp LM, van Es $\mathrm{IE}$, et al. Tie2 signaling cooperates with TNF to promote the proinflammatory activation of human macrophages independently of macrophage functional phenotype. PLoS One 2014;9:e82088.

41. Seok SH, Heo Jl, Hwang JH, Na YR, Yun JH, Lee EH, et al. Angiopoietin-1 elicits pro-inflammatory responses in monocytes and differentiating macrophages. Mol Cells 2013;35:550-556.

42. Kaczmarek A, Vandenabeele P, Krysko DV. Necroptosis: the release of damage-associated molecular patterns and its physiological relevance. Immunity 2013;38:209-223.

43. Wallach D, Kang TB, Kovalenko A. Concepts of tissue injury and cell death in inflammation: a historical perspective. Nat Rev Immunol 2014;14:51-59.

44. Yu B, Li X, Wan Q, Han W, Deng C, Guo C. High-mobility group box-1 protein disrupts alveolar elastogenesis of hyperoxia-injured newborn lungs. J Interferon Cytokine Res 2016;36:159-168.

45. Conos SA, Chen KW, De Nardo D, Hara H, Whitehead L, Núñez G, et al Active MLKL triggers the NLRP3 inflammasome in a cell-intrinsic manner. Proc Natl Acad Sci USA 2017;114:E961-E969.

46. Rodriguez DA, Weinlich R, Brown S, Guy C, Fitzgerald P, Dillon CP, et al. Characterization of RIPK3-mediated phosphorylation of the activation loop of MLKL during necroptosis. Cell Death Differ 2016; 23:76-88. 